\title{
Nonlinear interaction of charged particles with nonplane counterpropagating laser pulses of relativistic intensities
}

\author{
H. K. Avetissian* and Kh. V. Sedrakian \\ Centre of Strong Fields Physics, Yerevan State University, 1 A. Manukian, Yerevan 0025, Armenia
}

(Received 10 August 2010; published 14 October 2010)

\begin{abstract}
The nonlinear threshold phenomena of particle reflection and capture of electrons in the induced Compton process that have previously been revealed in the case of plane monochromatic counterpropagating waves, take place also with the actual nonplane laser pulses of ultrashort duration and lead to particle acceleration. In contrast to analogous phenomena in the induced Cherenkov and undulator processes, the Compton reflection-capture mechanism with laser pulses of relativistic intensities practically may be realized for arbitrary initial energies of particles. The acceleration effect for particles initially in rest is explored numerically, taking into account the significance of this case connected with the relativistic electron bunches of high densities, which currently may be realized by relativistic lasers on the ultrathin solid foils where the electrons initially are almost in rest.
\end{abstract}

DOI: 10.1103/PhysRevSTAB.13.101304

PACS numbers: 41.75.Jv, 41.75. Ht

\section{INTRODUCTION}

The significant advance in laser technology during the recent decade made available the ultrashort superintense laser pulses of relativistic intensities [1,2], which really create new nonlinear channels for diverse laser-particle interactions [3] and determine, in general, the future of laser accelerators [4-9] of superhigh energies, including laser-plasma accelerators [10-16].

In principle, the realization of an acceleration problem in any laser-induced process requires the possible largest coherent lengths for particle-laser stimulated interaction, i.e., one needs such a coherent process where due to the certain resonance the particle-wave interaction cross section enhances by several orders in magnitude [3]. On the other hand, in the coherent processes such as Cherenkov, Compton, and undulator ones, the nonlinear threshold phenomena of particle reflection and capture take place, which occur on the shortest interaction lengths, even shorter than a laser wavelength, because of the coherent/threshold feature of the nonlinear resonance in the above critical fields at which the interaction from an effective wave-barrier proceeds [3]. Consequently, the acceleration lengths due to the reflectioncapture phenomena are much less than the usual ones corresponding to common acceleration regimes in other processes. Hence, the laser acceleration process will be more effective in these coherent processes with the nonlinear resonances of threshold nature requiring shortest interaction lengths. The mentioned threshold phenomena of particle reflection capture in the induced Cherenkov process has been revealed for a plane transverse electromagnetic (e.m.) wave and in the Compton and undulator processes for monochromatic waves. For ultrashort nonplane laser pulses these phenomena with the subsequent

\footnotetext{
*avetissian@ysu.am
}

acceleration effect in the induced Cherenkov and undulator processes have been studied in the works $[17,18]$, respectively. However, in contrast to Cherenkov and undulator processes where practically relativistic/ultrarelativistic beams are needed for realization of nonlinear Cherenkov resonance in gaseous media [17], or nonlinear resonance in a magnetic undulator with the step of the order of a few $\mathrm{cm}$ [18], the considered here induced Compton mechanism for particle reflection capture and acceleration practically may be realized for arbitrary initial energies of the particles. In particular, using laser pulses of certain frequencies, one can accelerate initially nonrelativistic beams, or even particles in rest. Second, at the same frequencies of counterpropagating laser pulses in the induced Compton process one can achieve the cancellation of particle transverse momenta acquired in the field for bunches from the emerging relativistic electron targets (elimination of a bunch angular divergence), as it has been proposed in the paper [19] to generate uniform relativistic electron layers at the coherent Compton backscattering on the ultrathin solid foils (see also [20]).

In the present paper the nonlinear dynamics of charged particle interaction with the strong nonplane counterpropagating laser pulses of finite time-space configurations in vacuum is investigated to find out the influence of finite duration and transverse space sizes of real laser pulses on the reflection-capture phenomena in the induced Compton scattering and, consequently, the particle acceleration process.

\section{NONLINEAR DYNAMICS OF A CHARGED PARTICLE IN THE FIELD OF COUNTERPROPAGATING NONPLANE LASER PULSES IN VACUUM}

Let us consider the nonlinear dynamics of a charged particle in the induced Compton scattering process, i.e., the 
nonlinear interaction with the two counterpropagating strong laser beams of real time-space configurations in vacuum. As we are interested in superintense ultrashort focused laser pulses of relativistic intensities, for which the analytical solution of relativistic equations of motion of a particle is very complicated and we need the numerical integration of corresponding equations, then it is convenient to represent the particle's classical equations of motion in the form of dimensionless quantities:

$$
\begin{gathered}
\frac{d \boldsymbol{\Pi}}{d t^{\prime}}=\boldsymbol{\xi}_{1 E}\left(t^{\prime}, \mathbf{r}^{\prime}\right)+\frac{1}{\sqrt{1+\Pi^{2}}}\left[\boldsymbol{\Pi} \times \boldsymbol{\xi}_{1 H}\left(t^{\prime}, \mathbf{r}^{\prime}\right)\right] \\
+\frac{\omega_{2}}{\omega_{1}}\left(\xi_{2 E}\left(t^{\prime}, \mathbf{r}^{\prime}\right)+\frac{1}{\sqrt{1+\Pi^{2}}}\left[\boldsymbol{\Pi} \times \boldsymbol{\xi}_{2 H}\left(t^{\prime}, \mathbf{r}^{\prime}\right)\right]\right), \\
\frac{d \mathbf{r}^{\prime}}{d t^{\prime}}=\frac{\mathbf{\Pi}}{\sqrt{1+\Pi^{2}}} .
\end{gathered}
$$

Here the dimensionless momentum of the particle $\mathbf{I}$, time $t^{\prime}$, and coordinate $\mathbf{r}^{\prime}$ were introduced as follows:

$$
\boldsymbol{\Pi}=\frac{\mathbf{p}}{m c}, \quad t^{\prime}=\omega_{1} t, \quad \mathbf{r}^{\prime}=\frac{\omega_{1}}{c} \mathbf{r},
$$

and the dimensionless relativistic intensity parameters of e.m. waves,

$$
\begin{array}{cc}
\boldsymbol{\xi}_{1 E}=\frac{e \mathbf{E}_{1}}{m c \omega_{1}}, & \boldsymbol{\xi}_{2 E}=\frac{e \mathbf{E}_{2}}{m c \omega_{2}}, \\
\boldsymbol{\xi}_{1 H}=\frac{e \mathbf{H}_{1}}{m c \omega_{1}}, & \boldsymbol{\xi}_{2 H}=\frac{e \mathbf{H}_{2}}{m c \omega_{2}},
\end{array}
$$

characterize the electric (E) and magnetic (H) field strengths of the counterpropagating nonplane laser pulses of finite time-space (transversal) envelopes with the carrier frequencies $\omega_{1}$ and $\omega_{2}$ (let $\omega_{1}>\omega_{2}$ ), propagating along the axis $O X$ and in the opposite direction, respectively ( $e$ is the charge, $m$ is mass of the particle, and $c$ is the light speed in vacuum).

We will assume that the laser pulses are of circular polarizations with the electric field strength envelopes $E_{1}\left(y, z, t-\frac{x}{c}\right), E_{2}\left(y, z, t+\frac{x}{c}\right):$

$$
\begin{gathered}
\mathbf{E}_{1}(x, t)=\left[0, E_{10}\left(y, z, t-\frac{x}{c}\right) \cos \omega_{1}\left(t-\frac{x}{c}\right),\right. \\
\left.E_{10}\left(y, z, t-\frac{x}{c}\right) \sin \omega_{1}\left(t-\frac{x}{c}\right)\right], \\
\mathbf{E}_{2}(x, t)=\left[0, E_{20}\left(y, z, t+\frac{x}{c}\right) \cos \omega_{2}\left(t+\frac{x}{c}\right),\right. \\
\left.E_{20}\left(y, z, t+\frac{x}{c}\right) \sin \omega_{2}\left(t+\frac{x}{c}\right)\right] .
\end{gathered}
$$

The particle energy change in the field is given by the equation

$$
\frac{d \gamma}{d t^{\prime}}=\frac{\boldsymbol{\Pi}}{\sqrt{1+\Pi^{2}}} \cdot\left(\boldsymbol{\xi}_{1 E}\left(t^{\prime}, \mathbf{r}^{\prime}\right)+\frac{\omega_{2}}{\omega_{1}} \boldsymbol{\xi}_{2 E}\left(t^{\prime}, \mathbf{r}^{\prime}\right)\right)
$$

where $\gamma=\sqrt{1+\Pi^{2}}$ is the Lorentz factor.

Equations (1) and (2) allow the exact analytical solution in case of plane monochromatic waves of circular polarizations and if the particle initial velocity is directed along the axis of the waves propagation [3]. Thus, taking into account the relation between the electric and magnetic field strengths of transverse e.m. waves,

$$
\begin{gathered}
\boldsymbol{\xi}_{1 H}\left(t^{\prime}, \mathbf{r}^{\prime}\right)=\left[\hat{\mathbf{x}} \times \boldsymbol{\xi}_{1 E}\left(t^{\prime}, \mathbf{r}^{\prime}\right)\right] \\
\boldsymbol{\xi}_{2 H}\left(t^{\prime}, \mathbf{r}^{\prime}\right)=-\left[\hat{\mathbf{x}} \times \boldsymbol{\xi}_{2 E}\left(t^{\prime}, \mathbf{r}^{\prime}\right)\right]
\end{gathered}
$$

( $\hat{\mathbf{x}}$ is the unit vector along the $O X$ axis) in Eqs. (1) and (2), for a particle with an initial velocity $\mathrm{v}_{0}=\mathrm{v}_{0 x}$ we obtain from these equations the integral of motion in the induced Compton process:

$$
\gamma\left(t^{\prime}\right)-\frac{1}{n_{\mathrm{eff}}} \Pi_{x}\left(t^{\prime}\right)=\mathrm{const} ; \quad n_{\mathrm{eff}}=\frac{\omega_{1}+\omega_{2}}{\omega_{1}-\omega_{2}},
$$

with the help of which one can determine the particle energy in the field of a strong counterpropagating plane monochromatic waves [in fact to integrate the equations of motion (1) and (2)] [3]:

$$
\begin{aligned}
\gamma= & \frac{\gamma_{0}}{n_{\mathrm{eff}}^{2}-1}\left\{n_{\mathrm{eff}}^{2}\left(1-\frac{\mathrm{v}_{0}}{c n_{\mathrm{eff}}}\right)\right. \\
& \mp\left[\left(1-n_{\mathrm{eff}} \frac{\mathrm{v}_{0}}{c}\right)^{2}-\frac{n_{\mathrm{eff}}^{2}-1}{\gamma_{0}^{2}}\left\{\xi_{10}^{2}+\xi_{20}^{2}+2 \xi_{10} \xi_{20}\right.\right. \\
& \left.\left.\left.\times \cos \left[\left(1-\frac{\omega_{2}}{\omega_{1}}\right)\left(t^{\prime}-n_{\mathrm{eff}} x^{\prime}\right)\right]\right\}\right]^{1 / 2}\right\}
\end{aligned}
$$

Here the quantities $\gamma_{0}, \xi_{10}, \xi_{20}$ are the normalized initial energy of the particle and amplitudes of the plane monochromatic waves, respectively, and $n_{\text {eff }}$ is the refractive index of the slowed interference wave, formed by the two counterpropagating waves of different frequencies in the induced nonlinear Compton process, which causes the threshold phenomenon of particle reflection. Thus, according to formula (10), in the induced nonlinear Compton process there is a critical value of the effective wave intensity $\xi_{\text {cr }}$ (when the expression under the root becomes zero, which corresponds to the nonlinear Compton resonance in the field), above which $\xi_{\text {eff }} \equiv \xi_{10}+\xi_{20}>\xi_{\text {cr }}$, the root becomes complex, and then changes its sign corresponding to particle reflection from the slowed interference wave. Such inelastic reflection from the moving wave barrier is accompanied with the particle acceleration or deceleration, depending on the initial relative velocity of the particle with respect to the resonant value [3]. 


\section{NUMERICAL TREATMENT OF INDUCED COMPTON PROCESS WITH INTENSE LASER PULSES OF SHORT DURATION AND NONPLANE CONFIGURATIONS}

To clear up the influence of finite time-space effects of real ultrashort laser pulses of nonplane configurations and to reveal the mentioned above peculiarity in the dynamics of the induced nonlinear Compton process with the particle acceleration, the numerical integration of Eqs. (1), (2), and (8) with the Eqs. (6) and (7) has been made at first for the plane laser pulses of finite duration, neglecting temporarily the transversal space dependence of actual laser beams. We present the results of numerical treatment. The simulations have been made for laser pulses of plane form, described by the following envelopes of amplitudes in Eqs. (6) and (7):

$$
\xi_{1 E}(\tau)=\frac{\xi_{10}}{\cosh \left(\frac{\tau}{\tau_{0}}\right)}, \quad \xi_{2 E}(\eta)=\frac{\xi_{20}}{\cosh \left(\frac{\eta-\varphi_{0}}{\eta_{0}}\right)},
$$

where $\tau=x^{\prime}-t^{\prime}$ and $\eta=x^{\prime}+t^{\prime}$ are the wave dimensionless coordinates, $\tau_{0}, \eta_{0}$ are the normalized durations of counterpropagating laser beams with carrier frequencies $\omega_{1}, \omega_{2}$ and peak values of intensity parameters $\xi_{10}$ and $\xi_{20}$, respectively. Here the constant phase $\varphi_{0}$ in the second pulse is introduced to express the relative time delay between the counterpropagating pulses.

For exploration of acceleration effect due to the particle reflection-capture phenomena, we consider the parameters' initial values not greatly far from nonlinear resonance condition (in the field), at which the required critical intensities $\xi_{\text {cr }}$ are not too high (since for particle reflection and consequent acceleration it is necessary $\xi_{\text {eff }}>\xi_{\text {cr }}$ ). For that, the particle initial velocity $\mathrm{v}_{0 x}$ should not be much different from the phase velocity of the slowed interference wave $\mathrm{v}_{\mathrm{ph}}=c / n_{\text {eff }}$. The latter determines the optimal range of the particle initial energies for acceleration by laser pulses of moderate relativistic intensities $\xi \sim 1$ (for the initial energies greatly far from the resonant values $\xi_{\text {cr }} \gg$ 1 and laser sources of ultrarelativistic intensities are required; although for current state of the art such intensities are achievable with the focused ultrashort laser pulses of a few femtosecond durations). Thus, for initially nonrelativistic particles, particularly for the particles initially in rest, the waves' frequencies $\omega_{1}$ and $\omega_{2}$ which define the phase velocity of the slowed interference wave $c / n_{\text {eff }}$ must not be greatly different (practically $\omega_{1} / \omega_{2}$ may be varied for this propose from two to ten with existing nowadays lasers), i.e., for the nonlinear acceleration effect on the base of the reflection-capture phenomena in the induced Compton process we can use two counterpropagating laser beams, for example, in infrared-optical domain. The opposite case of $\omega_{1} \gg \omega_{2}$ (which corresponds to $n_{\text {eff }}-1 \ll 1$ ) can be used for acceleration of initially relativistic particles.

To reveal the particle reflection-capture phenomena and nonlinear acceleration effect in the induced Compton process with the real laser pulses, here we will consider the more interesting case of particles initially in rest, taking into account that relativistic electron bunches of high densities currently may be realized on the ultrathin solid foils where the electrons initially are almost in rest [19]. We will present the results of numerical integration of Eqs. (1), (2), and (8) with the Eqs. (6)-(11) for plane wave pulses of finite duration in two cases of $\omega_{1} / \omega_{2}=$ 10 (for example, with the $\lambda_{1}=1.06 \mu \mathrm{m}$ of $\mathrm{Nd}$ :YAG and $\lambda_{2}=10.6 \mu \mathrm{m}$ of $\mathrm{CO}_{2}$ lasers) and $\omega_{1} / \omega_{2}=2$ (e.g., with the $\lambda_{1}=0.53 \mu \mathrm{m}$ of Nd:YAG second harmonic and $\lambda_{2}=$ $1.06 \mu \mathrm{m}$ of Nd:YAG lasers). Concerning the intensities of laser beams, in all considered cases-both for plane pulses and nonplane-focused laser pulses-for the peak values of intensity parameters we assume $\xi_{10}=\xi_{20}=2$ at $\omega_{1} / \omega_{2}=10$, and $\xi_{10}=\xi_{20}=0.5$ at $\omega_{1} / \omega_{2}=2$ which corresponds to the above critical values.

The energy evolution of a particle initially in rest (at the position $x_{0}=0 ; y, z$ coordinates are cyclic in the plane waves because of space homogeneity in the plane $y z$ ) is plotted in the field of two counterpropagating laser pulses in vacuum. Figure 1(a) illustrates the particle energy dependence on the normalized time $t^{\prime}=\omega_{1} t$ for laser pulses of dimensionless durations $\tau_{0}=50, \eta_{0}=500$ in case $\omega_{1} / \omega_{2}=10$, at the relative initial phase between the pulses $\varphi_{0}=191$, which correspond to time duration of
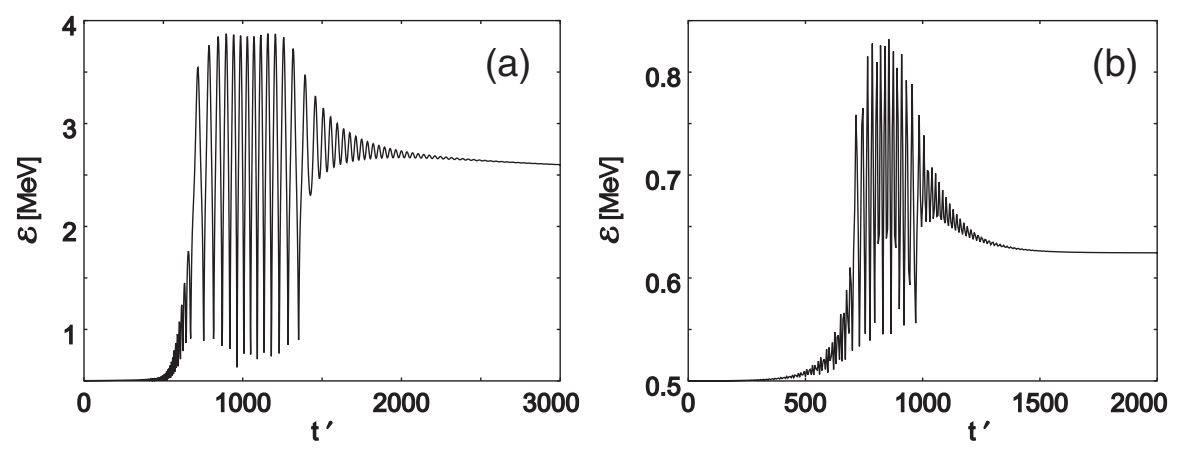

FIG. 1. Reflection capture of a particle initially in rest (at the longitudinal position $x_{0}=0$; transversal coordinates $y, z$ are cyclic for plane waves, so $y_{0}=z_{0}=0$ ) from a plane counterpropagating laser pulses of finite duration (a) for $\omega_{1} / \omega_{2}=10$, and (b) $\omega_{1} / \omega_{2}=2$. The laser intensities have above critical peak values $\xi_{10}=\xi_{20}=2$ for (a), and $\xi_{10}=\xi_{20}=0.5$ for (b). 
high $\left(\omega_{1}\right)$ frequency laser: $167 \mathrm{fs}$, low $\left(\omega_{2}\right)$ frequency laser: $1670 \mathrm{fs}$, and with the $637 \mathrm{fs}$ time delay of the low frequency pulse in respect to high frequency one. Figure 1 (b) corresponds to $\omega_{1} / \omega_{2}=2$, at $\tau_{0}=100, \eta_{0}=200$, and $\varphi_{0}=5$. For these values of parameters the peak value of the resulting wave intensity is above the critical point and, as Fig. 1 shows, the energy of the particle initially in rest is changed abruptly due to the reflection capture in the induced Compton process, and after the reflection from a corresponding phase plane of the slowed wave-pulse intensity envelope acquires $\mathcal{E} \simeq 2.5 \mathrm{MeV}$ energy at $\omega_{1} / \omega_{2}=10$, and $\mathcal{E} \gtrsim 0.6 \mathrm{MeV}$ at $\omega_{1} / \omega_{2}=2$.
Figure 2 illustrates the evolution of the particle (bunch) energies with different initial positions $x_{0}$ along the axis of laser propagation, at the same values of the other parameters, mentioned in the text for Fig. 1.

To explore the nonlinear dynamics of the considered process for real nonplane-focused laser pulses of relativistic intensities, we will integrate numerically the particle equations of motion [Eqs. (1), (2), and (8)] for energy in the field of two counterpropagating focused laser pulses of circular polarizations, the approximate nonplane forms of which may be modeled in the following form (for a linear polarization see [21], and for a circular one [18]):

$$
\begin{aligned}
E_{1 x}= & E_{10} g_{1}(\tau) \frac{\lambda_{1} w_{10}^{2} e^{-r_{\perp}^{2} / w_{1}^{2}(x)}}{\pi w_{1}^{4}(x)}\left\{\left[-2 \frac{x y}{x_{1 R}}+\left(1-\frac{x^{2}}{x_{1 R}^{2}}\right) z\right] \cos \left(\tau+\frac{r_{\perp}^{2} x}{x_{1 R} w_{1}^{2}(x)}\right)\right. \\
& \left.+\left[\left(1-\frac{x^{2}}{x_{1 R}^{2}}\right) y+2 \frac{x z}{x_{1 R}}\right] \sin \left(\tau+\frac{r_{\perp}^{2} x}{x_{1 R} w_{1}^{2}(x)}\right)\right\}, \\
E_{1 y}= & E_{10} g_{1}(\tau) \frac{w_{10}^{2} e^{-r_{\perp}^{2} / w_{1}^{2}(x)}}{w_{1}^{2}(x)}\left\{\cos \left(\tau+\frac{r_{\perp}^{2} x}{x_{1 R} w_{1}^{2}(x)}\right)+\frac{x}{x_{1 R}} \sin \left(\tau+\frac{r_{\perp}^{2} x}{x_{1 R} w_{1}^{2}(x)}\right)\right\}, \\
E_{1 z}= & -E_{10} g_{1}(\tau) \frac{w_{10}^{2} e^{-r_{\perp}^{2} / w_{1}^{2}(x)}}{w_{1}^{2}(x)}\left\{-\frac{x}{x_{1 R}} \cos \left(\tau+\frac{r_{\perp}^{2} x}{x_{1 R} w_{1}^{2}(x)}\right)+\sin \left(\tau+\frac{r_{\perp}^{2} x}{x_{1 R} w_{1}^{2}(x)}\right)\right\}, \\
H_{1 x}= & E_{10} g_{1}(\tau) \frac{\lambda_{1} w_{10}^{2} e^{-r_{\perp}^{2} / w_{1}^{2}(x)}}{\pi w_{1}^{4}(x)}\left\{\left[-2 \frac{x z}{x_{1 R}}-\left(1-\frac{x^{2}}{x_{1 R}^{2}}\right) y\right] \cos \left(\tau+\frac{r_{\perp}^{2} x}{x_{1 R} w_{1}^{2}(x)}\right)\right. \\
& \left.+\left[\left(1-\frac{x^{2}}{x_{1 R}^{2}}\right) z-2 \frac{x y}{x_{1 R}}\right] \sin \left(\tau+\frac{r_{\perp}^{2} x}{x_{1 R} w_{1}^{2}(x)}\right)\right\}, \\
H_{1 y}= & -E_{1 z} ; \quad H_{1 z}=E_{1 y},
\end{aligned}
$$

where $r_{\perp}^{2}=y^{2}+z^{2}, w_{1}(x)=w_{10} \sqrt{1+x^{2} / x_{1 R}^{2}}, x_{1 R}=\pi w_{10}^{2} / \lambda_{1}$ is the Rayleigh length for high frequency (of wavelength $\left.\lambda_{1}=2 \pi c / \omega_{1}\right)$ focused laser pulse with the waist $w_{10}$ in the focal plane $x=0$, and $g_{1}(\tau)=1 / \cosh \left(\tau / \tau_{0}\right)$.

For electric and magnetic fields of a low frequency (of wavelength $\lambda_{2}=2 \pi c / \omega_{2}$ ) focused laser pulse propagating in the opposite direction to the high frequency pulse, we have

$$
\begin{aligned}
E_{2 x}= & -E_{20} g_{2}(\eta) \frac{\lambda_{2} w_{20}^{2} e^{-r_{\perp}^{2} / w_{2}^{2}(x)}}{\pi w_{2}^{4}(x)}\left\{\left[2 \frac{x y}{x_{2 R}}+\left(1-\frac{x^{2}}{x_{2 R}^{2}}\right) z\right] \cos \left(\eta+\frac{r_{\perp}^{2} x}{x_{2 R} w_{2}^{2}(x)}\right)\right. \\
& \left.-\left[\left(1-\frac{x^{2}}{x_{2 R}^{2}}\right) y-2 \frac{x z}{x_{2 R}}\right] \sin \left(\eta+\frac{r_{\perp}^{2} x}{x_{2 R} w_{2}^{2}(x)}\right)\right\}, \\
E_{2 y}= & E_{20} g_{2}(\eta) \frac{w_{20}^{2} e^{-r_{\perp}^{2} / w_{2}^{2}(x)}}{w_{2}^{2}(x)}\left\{\cos \left(\eta+\frac{r_{\perp}^{2} x}{x_{2 R} w_{2}^{2}(x)}\right)+\frac{x}{x_{2 R}} \sin \left(\eta+\frac{r_{\perp}^{2} x}{x_{2 R} w_{2}^{2}(x)}\right)\right\}, \\
E_{2 z}= & -E_{20} g_{2}(\eta) \frac{w_{20}^{2} e^{-r_{\perp}^{2} / w_{2}^{2}(x)}}{w_{2}^{2}(x)}\left\{\frac{x}{x_{2 R}} \cos \left(\eta+\frac{r_{\perp}^{2} x}{x_{2 R} w_{2}^{2}(x)}\right)-\sin \left(\eta+\frac{r_{\perp}^{2} x}{x_{2 R} w_{2}^{2}(x)}\right)\right\}, \\
H_{2 x}= & E_{20} g_{2}(\eta) \frac{\lambda_{2} w_{20}^{2} e^{-r_{\perp}^{2} / w_{2}^{2}(x)}}{\pi w_{2}^{4}(x)}\left\{\left[2 \frac{x z}{x_{2 R}}-\left(1-\frac{x^{2}}{x_{2 R}^{2}}\right) y\right] \cos \left(\eta+\frac{r_{\perp}^{2} x}{x_{2 R} w_{2}^{2}(x)}\right)\right. \\
& \left.-\left[\left(1-\frac{x^{2}}{x_{2 R}^{2}}\right) z+2 \frac{x y}{x_{2 R}}\right] \sin \left(\eta+\frac{r_{\perp}^{2} x}{x_{2 R} w_{2}^{2}(x)}\right)\right\}, \\
H_{2 y}= & E_{2 z} ; \quad H_{2 z}=-E_{2 y},
\end{aligned}
$$



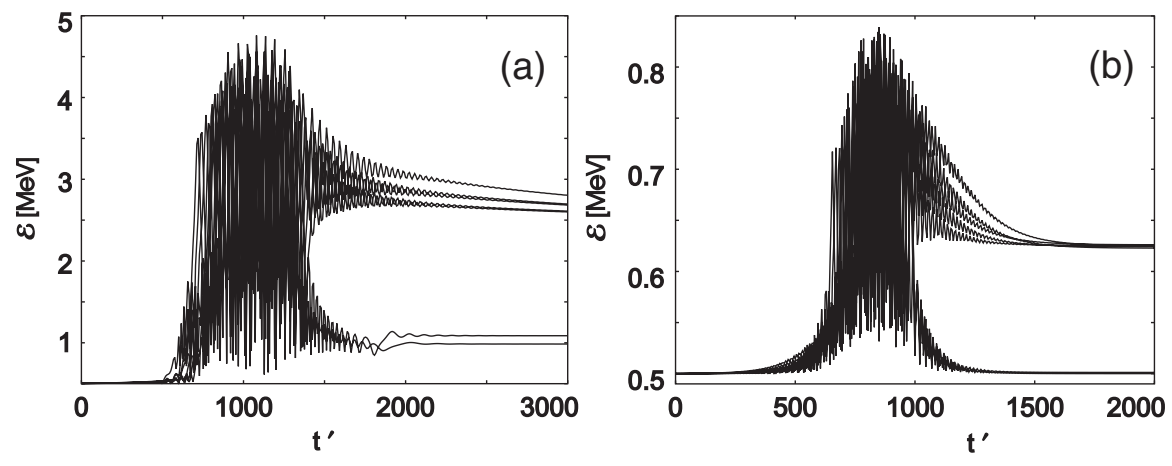

FIG. 2. The particle energy evolution in the field of two counterpropagating plane laser pulses and after the interaction corresponding to reflection-capture phenomena depended on the particle initial longitudinal positions $x_{0}$ (bunch initially in rest): (a) $x_{0} \in\left[0 ; 30 \lambda_{1}\right]$ (with the step $5 \lambda_{1}$ ) for $\omega_{1} / \omega_{2}=10$, and (b) $x_{0} \in\left[-15 \lambda_{1} ; 15 \lambda_{1}\right]$ (step $5 \lambda_{1}$ ) for $\omega_{1} / \omega_{2}=2$. Other parameters are the same as noted in the text for Fig. 1 .

where $w_{2}(x)=w_{20} \sqrt{1+x^{2} / x_{2 R}^{2}}, \quad x_{2 R}=\pi w_{20}^{2} / \lambda_{2}$ is the Rayleigh length for a focused pulse with the waist $w_{20}$ (in the focal plane $x=0$ ), and $g_{2}(\eta)=1 / \cosh \left(\eta / \eta_{0}\right)$.

The integration of the relativistic equations of motion of a particle [Eqs. (1) and (2)] in the given field of the counterpropagating waves has been made with the Runge-Kutta method [22] (the precision of numerical simulations is justified by the known fourth-order adaptive Runge-Kutta method). The results of numerical integration of particle equations of motion and for energy in the field (12) and (13) are presented in Figs. 3-6. The graphics of particle energy evolution in the nonlinear induced Compton process with focused laser pulses of ultrashort duration, illustrated in Figs. 1-3, prove the particle
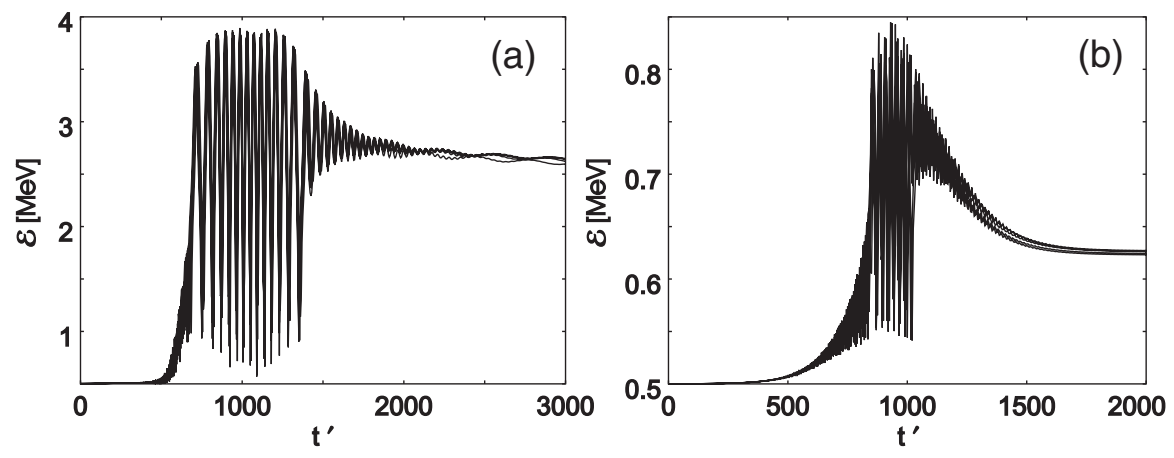

FIG. 3. Reflection capture of the particles from the nonplane counterpropagating laser pulses at different initial longitudinal positions $x_{0}\left(y_{0}=z_{0}=0\right.$ ): (a) $x_{0} \in\left[-\lambda_{1} ; \lambda_{1}\right]$ (with the step $\left.0.5 \lambda_{1}\right)$ for $\omega_{1} / \omega_{2}=10$, and (b) $x_{0} \in\left[-0.4 \lambda_{1} ; 0.4 \lambda_{1}\right]$ (step $0.2 \lambda_{1}$ ) for $\omega_{1} / \omega_{2}=2$. Other parameters for focused nonplane laser pulses are defined in the text for Fig. 3 .
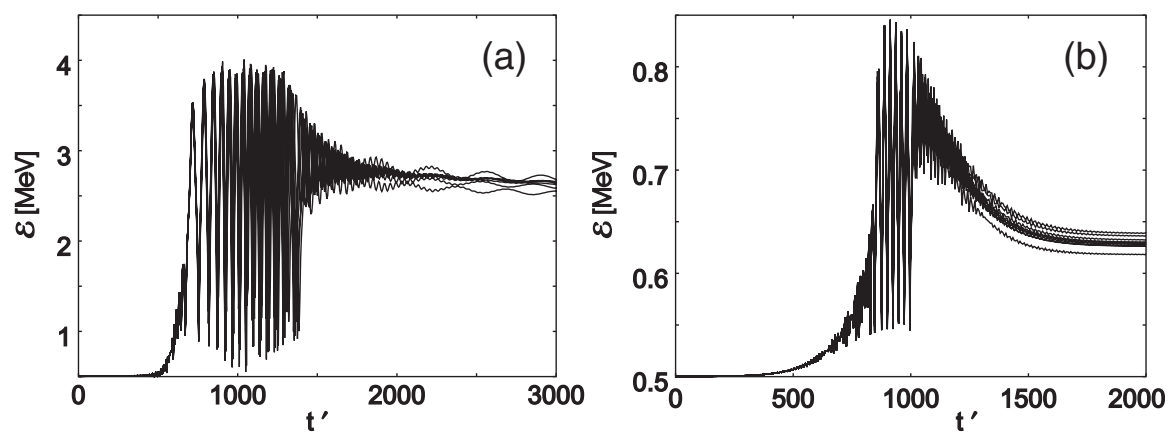

FIG. 4. The dependence of the particle energy on the initial transversal positions $\left(y_{0}\right)$ in the reflection-capture regime by two nonplane counterpropagating laser pulses: (a) $y_{0} \in\left[0 ; 3 \lambda_{1}\right]$ (with the step $0.5 \lambda_{1}$ ) for $\omega_{1} / \omega_{2}=10$, and (b) $y_{0} \in\left[0 ; 4 \lambda_{1}\right]$ (step $0.5 \lambda_{1}$ ) for $\omega_{1} / \omega_{2}=2$. Here longitudinal coordinate $x_{0}=0$, other parameters are the same as for Fig. 3 . 


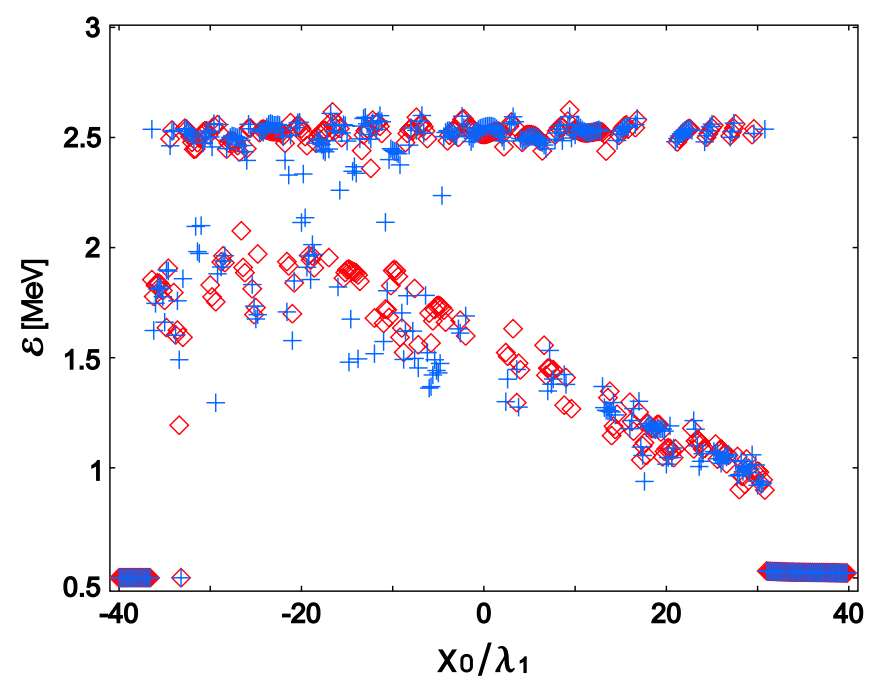

FIG. 5. Particle energy dependence on the initial longitudinal positions $x_{0}$ (normalized) at the reflection capture both by the plane (red small square symbols) and nonplane-focused (blue crisscross symbols) laser pulses under the same condition for $\omega_{1} / \omega_{2}=10$; other parameters are defined in the text for Figs. 1 and 3.

reflection-capture phenomena by real nonplane e.m. wave pulses of arbitrary duration.

In numerical simulations for focused laser pulses we assumed the following transverse space sizes in the focal plane (pulse waist): $w_{10}=100 \lambda_{1}$ and $w_{20}=200 \lambda_{1}$ for high and low frequency lasers, respectively (in Figs. 3-6 the values of $w_{20}$ in scale of $\lambda_{2}$ are $w_{20}=20 \lambda_{2}$ for the case $\omega_{1} / \omega_{2}=10$, and $w_{20}=100 \lambda_{2}$ for $\omega_{1} / \omega_{2}=2$ ).

Figure 4 demonstrates the case of interaction when the particles initially situated at different distances from the laser beam axis.

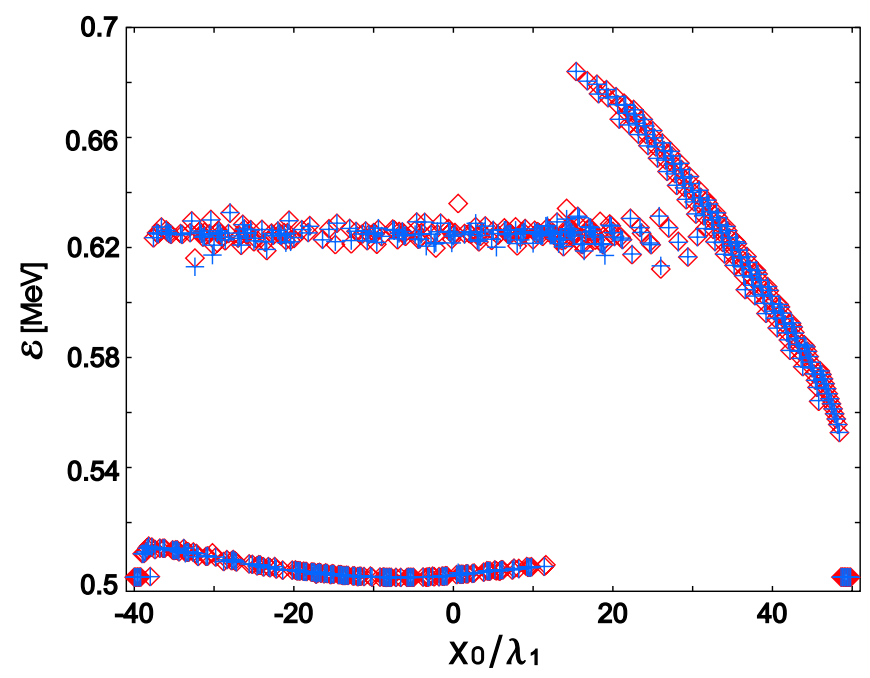

FIG. 6. The same dependence as in Fig. 5 for $\omega_{1} / \omega_{2}=2$; other parameters are defined in the text for Figs. 1 and 3.
To show the influence of space effects because of nonplane fields of focused ultrapower laser pulses on the nonlinear dynamics of induced Compton process and acceleration effect due to the considered threshold phenomena, we present for comparison the graphics of particle energy dependence on the initial positions at the reflection capture, both by the plane and nonplane-focused laser pulses under the same condition, which are demonstrated in Figs. 5 and 6: for cases $\omega_{1} / \omega_{2}=10$ and $\omega_{1} / \omega_{2}=2$, respectively. As is seen from these graphics, for the considered parameters of laser beams the reflection capture and, consequently, acceleration effect occurs for the particles initially situated about in the range $x_{0} \in$ $\left[-40 \lambda_{1} ; 40 \lambda_{1}\right]$; the other particles remain with the initial energy $\mathcal{E}_{0}=m c^{2}$, since the threshold condition for reflection capture $\xi_{\text {eff }}>\xi_{\text {cr }}$ is not satisfied for those particles (because of smaller intensity values on the envelope-profile wings of ultrashort laser pulses).

Apart from the mentioned peculiarities in the considered process, we see that the acceleration effect due to the particle reflection capture at the induced nonlinear Compton scattering on the nonplane counterpropagating focused laser pulses neither depends on the field magnitudes (only should be the above threshold field: $\xi_{\text {eff }}>\xi_{\text {cr }}$ ) nor on the interaction length (the latter-reflection length-may be smaller than a laser wavelength). This fact opens a principally new way for laser acceleration of charged particles on the ultrashort distances in vacuum using shortest (at present, a few femtosecond) superpower laser pulses of ultrarelativistic intensities $(\xi \gg 1)$ in the induced Compton process.

\section{CONCLUSION}

We present the results of the theoretical and numerical treatment of charged particle nonlinear interaction with the two nonplane counterpropagating laser pulses of relativistic intensities in vacuum corresponding to induced nonlinear Compton scattering. It is shown that the nonlinear threshold phenomena of particle reflection and capture, which have previously been revealed in the field of the plane monochromatic waves, take place also in the nonplane counterpropagating laser pulses of ultrashort duration and lead to particle acceleration. Such acceleration from the moving wave barrier (formed due to the nonlinear coherent interaction of a charged particle with the counterpropagating waves at the intensities above a certain critical value) occurs on the shortest interaction lengths, even shorter than a laser wavelength. So, the reflection-capture phenomena open a principally new way for laser acceleration of charged particles on the ultrashort distances in vacuum using shortest (at present, a few femtosecond) superpower laser pulses of ultrarelativistic intensities in the induced Compton process. On the other hand, in contrast to analogous induced coherent processes (Cherenkov and undulator) where the realization of the 
reflection-capture phenomena actually require an initial relativism, the considered Compton mechanism is applicable practically for arbitrary initial energies of particles, in particular, for initially nonrelativistic beams or even for particles in rest. The latter is of interest taking into account that relativistic electron bunches of high densities currently may be realized by relativistic lasers on the ultrathin solid foils where the electrons initially are almost in rest, and therefore this case is explored numerically with presentation of corresponding graphics.

\section{ACKNOWLEDGMENTS}

This work was supported by International Science and Technology Center (ISTC) Project No. A-1307.

[1] C. N. Danson et al., Nucl. Fusion 44, S239 (2004).

[2] G. A. Mourou, T. Tajima, and S. V. Bilanov, Rev. Mod. Phys. 78, 309 (2006).

[3] H. K. Avetissian, Relativistic Nonlinear Electrodynamics (Springer, New York, 2006).

[4] K. Nakajima et al., Phys. Rev. Lett. 74, 4428 (1995).

[5] P. Mora and B. Quesnel, Phys. Rev. Lett. 80, 1351 (1998).

[6] K. T. McDonald, Phys. Rev. Lett. 80, 1350 (1998).

[7] K. Nakajima, Laser Part. Beams 18, 519 (2000).
[8] P.X. Wang et al., Appl. Phys. Lett. 78, 2253 (2001).

[9] Ch. Varin, M. Piche, and M. A. Porras, Phys. Rev. E 71, 026603 (2005).

[10] R. Wagner, S.-Y. Chen, A. Maksimchuk, and D. Umstadter, Phys. Rev. Lett. 78, 3125 (1997).

[11] T. Tajima and J. M. Dawson, Phys. Rev. Lett. 43, 267 (1979).

[12] E. Esarey et al., IEEE Trans. Plasma Sci. 24, 252 (1996); IEEE J. Quantum Electron. 33, 1879 (1997).

[13] Th. Stohlker et al., Phys. Rev. Lett. 86, 983 (2001).

[14] M. Drescher et al., Sci. Exp. 291, 1923 (2001).

[15] A. Pukhov and J. Meyer-ter-Vehn, Appl. Phys. B 74, 355 (2002).

[16] S. V. Tochitsky et al., Phys. Rev. Lett. 92, 095004 (2004).

[17] H. K. Avetissian, S. S. Israelyan, and Kh. V. Sedrakian, Phys. Rev. ST Accel. Beams 10, 071301 (2007).

[18] H. K. Avetissian and Kh. V. Sedrakian, Phys. Rev. ST Accel. Beams13, 081301 (2010).

[19] H.-C. Wu, J. Meyer-ter-Vehn, J. Fernández, and B. M. Hegelich, Phys. Rev. Lett. 104, 234801 (2010).

[20] J. Meyer-ter-Vehn and H.-C. Wu, Eur. Phys. J. D 55, 433 (2009); H.-C. Wu and J. Meyer-ter-Vehn, Eur. Phys. J. D 55, 443 (2009).

[21] K. T. McDonald, arXiv:physics/0003056v2.

[22] W. H. Press, S. A. Teukolsky, W. T. Vetterling, and B.P. Flannery, Numerical Recipes in C (Cambridge University Press, Cambridge, U.K., 1992). 